\title{
A prospective study on contrast-enhanced magnetic resonance imaging of testicular lesions: distinctive features of Leydig cell tumours
}

\author{
Lucia Manganaro ${ }^{1} \cdot$ Valeria Vinci $^{1}$ - Carlotta Pozza ${ }^{2}$ - Matteo Saldari ${ }^{1}$. \\ Daniele Gianfrilli ${ }^{2}$ - Riccardo Pofi ${ }^{2}$ - Silvia Bernardo ${ }^{1}$ - Vito Cantisani ${ }^{1}$ - Andrea Lenzi ${ }^{2}$. \\ Michele Scialpi $^{3} \cdot$ Carlo Catalano $^{1} \cdot$ Andrea M. Isidori $^{2}$
}

Received: 11 January 2015 /Revised: 1 March 2015 / Accepted: 2 April 2015

(C) European Society of Radiology 2015

\begin{abstract}
Objectives Up to $20 \%$ of incidentally found testicular lesions are benign Leydig cell tumours (LCTs). This study evaluates the role of contrast-enhanced magnetic resonance imaging (MRI) in the identification of LCTs in a large prospective cohort study.

Materials and methods We enrolled 44 consecutive patients with at least one solid non-palpable testicular lesion who underwent scrotal MRI. Margins of the lesions, signal intensity and pattern of wash-in and wash-out were analysed by two radiologists. The frequency distribution of malignant and benign MRI features in the different groups was compared by using the chi-squared or Fisher's exact test. Sensitivity, specificity, positive and negative predictive value, and diagnostic accuracy were calculated.

Results The sensitivity of scrotal MRI to diagnose LCTs was $89.47 \%$ with $95.65 \%$ specificity; sensitivity for malignant lesions was $95.65 \%$ with $80.95 \%$ specificity. A markedly hypointense signal on T2-WI, rapid and marked wash-in followed by a prolonged washout were distinctive features significantly associated with LCTs. Malignant lesions were significantly associated with blurred margins, weak
\end{abstract}

Lucia Manganaro

lucia.manganaro@uniroma1.it

1 Department of Radiology, Sapienza University of Rome, Viale Regina Elena 324, 00161 Rome, Italy

2 Department of Experimental Medicine, Sapienza University of Rome, Viale del Policlinico 155, 00161 Rome, Italy

3 Department of Surgical and Biomedical Sciences, Division of Radiology 2, Perugia University, S. Maria della Misericordia Hospital, S. Andrea delle Fratte, 06156 Perugia, Italy hypointense signal on T2-WI , and weak and progressive wash-in. The overall diagnostic accuracy was $93 \%$.

Conclusions LCTs have distinctive contrast-enhanced MRI features that allow the differential diagnosis of incidental testicular lesions.

Key Points

- MRI is able to characterize testicular lesions suggesting a specific diagnosis.

- Rapid and marked wash-in is a common feature of Leydig cell tumours.

- Markedly hypointense T2-WI signal is significantly correlated with benign lesions.

- Blurred margins and weak hypointense T2-WI signal are correlated with malignant tumours.

- Weak and progressive wash-in features are present in $85 \%$ of seminomatous lesions.

Keywords Magnetic resonance imaging - Testicular cancer . Leydig cell tumours $\cdot$ Contrast enhanced MRI $\cdot$ Seminoma

\section{Introduction}

Testicular tumours account for $4-6 \%$ of all neoplasms of the male urogenital tract and for $1-2 \%$ of all cancers in young males [1]. A high proportion of small testicular lesions have been proven benign [2,3]. Leydig cell tumours account for up to $22 \%$ of tumours less than $1.5 \mathrm{~cm}$ in size, incidentally found during routine ultrasound (US) [2].

The ability to distinguish benign from malignant nonpalpable lesions pre-operatively allows opting for conservative surgery or follow-up in selected cases, with preservation of fertility, endocrine function and relevant savings for health services [4]. 
Scrotal US is the preferred modality in the assessment of male reproductive problems increasing the detection of incidentally non-palpable lesions. However, it does not allow a characterization of their nature $[5,6]$. A significant improvement in the diagnostic accuracy has been demonstrated by integrating US with contrast-enhanced US (CEUS) [2, 7, 8] and elastosonography [7, 9].

The issue is of particular relevance for monorchid patients and those with bilateral lesions.

Magnetic resonance imaging (MRI) has been recently proposed as an additional diagnostic procedure in the characterization of testicular masses, offering higher sensitivity and specificity than unenhanced US for selected testicular pathologies [10].

The role of MRI in the differential diagnosis of testicular masses, particularly the use of contrast agents, is still controversial. Although dynamic contrast-enhanced subtraction MRI (DCE-MRI) imaging can provide information about testicular perfusion by means of testicular contrast enhancement [11], the clinical value and diagnostic performance of DCEMRI is not yet established, as was recently demonstrated with CEUS [12].

The aim of this study was to evaluate prospectively the accuracy of qualitative and semi-quantitative contrast-enhanced MRI in the differential diagnosis of non-palpable testicular masses.

\section{Materials and methods}

\section{Patients}

The local review board approved the protocol, and all patients provided written informed consent. This was a prospective diagnostic accuracy study, conducted at our hospital, between 2006 and June 2012.

All patients included in the CEUS diagnostic study by Isidori et al. [2] with at least one solid, non-palpable testicular lesion, were offered undergoing MRI imaging of the scrotum with gadolinium enhancement. Indications for CEUS are described in Table 1. Only patients with proven histology (reference diagnosis) were considered in the current analysis.

Subjects underwent routine blood tests, hormonal investigations (LH, FSH, testosterone, inhibin B, SHBG, E2) and tumour markers (beta-HCG, alfa-FP, CEA, LDH, PLAP), and were scheduled to undergo scrotal MRI. Thereafter tissue-sparing surgical enucleation was offered for histological confirmation to all patients, independently of the CEUS and MRI results. Malignant tumours were immediately or subsequently treated with radical orchiectomy.

The time interval between CEUS, MRI and surgery was less than 3 weeks. The exclusion criteria were: absence of internal enhancement after contrast administration on MRI
Table 1 Reason for referral for scrotal ultrasound and MRI

\begin{tabular}{ll}
\hline Reason for scrotal ultrasound and MRI & $\begin{array}{l}\text { No. (\%) of patients, } \\
\mathrm{n}=44 \mathrm{n}=115\end{array}$ \\
\hline Infertility & $26(59.1)$ \\
Andrological screening/other & $6(13.6)$ \\
Testicular pain & $4(9.1)$ \\
$\begin{array}{l}\text { Previous orchiectomy for } \\
\text { contralateral tumour }\end{array}$ & $6(13.6)$ \\
Klinefelter syndrome & $1(2.3)$ \\
Hypogonadism & $1(2.3)$ \\
\hline
\end{tabular}

${ }^{\text {a }}$ Six patients had previous surgery of the testis for testicular tumour; this was the first scan for the remaining 38 patients

and absence of histological diagnosis. None had cardiac insufficiency.

\section{MRI protocol}

Patients did not require any preparation, except measuremnet of serum creatinine prior to administration of intravenous medium contrast. All patients were examined in the supine position. The penis was draped to the anterior abdominal wall. Testes were placed at a similar distance, with a towel placed between them. All the examinations were performed on a 1.5 T system (Siemens Magnetom Avanto, Erlangen, Germany), with the use of a phased array multichannel (32 channels) body coil. The MRI protocol included: orientation sequences: T2-weighted (T2-W) HASTE (Half Fourier Single Shot Turbo Spin Echo) (slice thickness $(\mathrm{SL})=6 \mathrm{~mm}$; repetition time $(\mathrm{TR})=1000 \mathrm{msec}$; echo time $(\mathrm{TE})=85 \mathrm{msec}$; flip angle $(F A)=150)$; morphological sequences: T2-W Turbo Spin Echo High resolution sequences $(\mathrm{SL}=3.00 \mathrm{~mm}$; $\mathrm{TR}=3659$ $5740 \mathrm{msec}$; TE=95-110 msec; $\mathrm{FA}=40-150$; field of view $(\mathrm{FOV})=240 \times 240 ;$ Matrix $=256 \times 224) ;$ T1-weighted $(\mathrm{T} 1-$ W) Flash 2D sequences (Fast low-angle single shot Gradient-Echo) with breath hold, with and without fat saturation $(\mathrm{SL}=5.5 \mathrm{~mm}$; $\mathrm{TR}=137-240 \mathrm{msec}$; $\mathrm{TE}=5 \mathrm{msec}$; FA 70; Matrix $=256 \times 205 ; \mathrm{FOV}=350 \times 350$ ); T1-WI in and out of phase $(\mathrm{SL}=3 \mathrm{~mm} ; \mathrm{TR}=125 \mathrm{msec} ; \mathrm{TE}=2-5 \mathrm{msec} ; \mathrm{FA}=70$; Matrix $=$ $256 \times 243 ; \mathrm{FOV}=450 \times 450)$.

A medium contrast agent (Gadobenate Dimeglumine, Multihance Bracco 0,1 mL/kg) was administered intravenously; we applied VIBE (volumetric interpolated breath-hold examination) sequences ( $\mathrm{SL}=4 \mathrm{~mm} ; \mathrm{FA}=12 ; \mathrm{FOV}=280 \times 280$; Matrix $=256 \times 224 ; \mathrm{TR}=4 \mathrm{msec} ; \mathrm{TE}=2 \mathrm{msec}$ ) in a dynamic protocol acquiring a pre-contrast scan followed by a postcontrastoghraphic series of six consecutives phases of $20 \mathrm{~s}$ each. Finally we completed the study with the elaboration of pre- and post-contrast sequences thus obtaining subtracted images. Examination was completed with a coronal plane scan of the abdomen in order to identify associated 
pathologies. The total acquisition time of the examination was $30 \mathrm{~min}$.

\section{Image analysis}

Radiologists used the Sony LMD-2451 MD monitor (resolution 1220 x 1920 pixels) for the prospective reading of mass lesion characteristics. MRI image interpretation was performed by two radiologists with more than 15 years and 5 years of experience in genitourinary imaging, respectively, blinded to the surgical or histopathological results, and discrepancies were resolved by consensus. Lesions were characterized on the basis of pre-contrast visualization of the lesion, wash-in and wash-out at semi-quantitative analysis, and pattern of contrast-enhancement.

Signal intensity with respect to normal testicular parenchyma on T2-weighted images (T2-WI) (markedly hypointense, slightly hypointense, isointense and slightly hyperintense), signal intensity on phase T1-weighted images (T1-WI) (hypointense, isointense, slightly hyperintense), and margins of the lesions (well-defined, blurred or irregular) were evaluated.

The time-signal intensity curve (TIC) from contrastenhanced MRI was obtained by manually placing a region of interest (ROI) within the lesions with care to exclude areas of haemorrhage and necrosis and with the aid of corresponding T1-WI and T2-WI; an identical ROI was placed on the adjacent parenchyma.

According to the shape of the TICs, the wash-in pattern was categorized as rapid or marked (enhancement peak within $1 \mathrm{~min}$ after agent injection), reflecting a high degree of vascularisation, and weak and progressive (when the previous condition did not occur).

The wash-out pattern was divided into rapid (decline abrupt of the signal intensity), slow and delayed (progressive decline of signal intensity), and absent (persistent or progressive enhancement, with continuous increase in signal intensity).

Contrast enhancement was classified as homogeneous, non-homogeneous or rim enhancement.

For each patient, on T2-WI the observers subjectively scored the degree of lesion-to-testis contrast according to the following five-point scale: $1=$ signal intensity of lesion is lower than those of surrounding normal testis; $2=$ signal intensity of lesion is equal to those of surrounding normal testis; $3=$ signal intensity of lesion is slightly higher than those of surrounding normal testis; $4=$ signal intensity of lesion is noticeably higher than those of surrounding normal testis; 5=signal intensity of lesion is markedly higher than those of surrounding normal testis. The mean visual scores for lesion-to-testis contrast was then calculated and compared.

\section{Statistical analysis}

Groups were compared using odds ratio (OR) confidence intervals for categorical variables. The frequency distribution of malignant and benign MRI features in the different group was compared by using the chi-squared or Fisher's exact test. Subgroup analysis was performed to limit the comparison to the largest groups of histotypes. Sensitivity, specificity and diagnostic accuracy of MRI findings in the assessment of benign and malignant testicular lesions were calculated.

Interobserver agreement was analysed by means of Kappa statistics. A positive correlation was considered to be indicated by a $\mathrm{k}$ value greater than 0 , poor correlation by values of $0.00-0.01$, low correlation by values of $0.01-0.20$, moderate correlation by values of $0.21-0.40$, good correlation by values of $0.41-0.60$, substantial correlation by values of $0.61-0.80$, and almost perfect agreement by values greater than 0.81 [13].

\section{Results}

\section{Clinical data}

Of the 115 patients who presented a non-palpable testicular lesion, 69 agreed to undergo a gadolinium-enhanced scrotal MRI. Of these, 25 patients were excluded because they showed no internal enhancement at MRI (6/69) or refused to undergo surgery (19/69) and thus no definite histology was available [2]. Therefore the final study group consisted of 44 patients (Fig. 1). The reason for referral is reported in Table 1.

Twenty-three patients had malignant tumours (20 seminomas, two embryonal carcinomas and one mixed germ cell tumour) and 21 had benign tumours (19 Leydig cell tumours and two Sertoli cell tumours). All 44 subjects underwent enucleation followed by radical orchiectomy in all 23 malignancies. The malignancies tended to be larger than benign tumours (mean diameter $0.9 \pm 0.31 \mathrm{~cm}$ vs. $0.6 \pm$ $0.28 \mathrm{~cm}$, respectively, $\mathrm{p}<0.001$; Table 2). Full hormonal data were available for 20 of the 44 patients. Of these, $34 \%$ of the patients had a FSH level of more than $7 \mathrm{mIU} / \mathrm{mL}(16.8 \pm 17.0$, normal range 1.38-9.58), $13 \%$ had a decreased testosterone level $(4.4 \pm 1.6 \mathrm{ng} / \mathrm{mL}$, normal range $2.8-11)$ and $20.5 \%$ had an elevated $\mathrm{LH}$ level $(8.5 \pm 8.4 \mathrm{mIU} / \mathrm{mL}$, normal range 1.8 8.16). Inhibin B, SHBG, E2 and tumour markers were normal at baseline in all 44 patients.

\section{MRI qualitative results}

Most of the histologically proven benign lesions appeared hypointense on T2-WI compared to malignant lesions (17/ $21,81 \%$, vs. $8 / 23,34.8 \%, \chi 29.537, p=0.03)$. The remaining benign lesions appeared slightly hypointense (14.3\% vs. $52.2 \%, \chi 27.013, \mathrm{p}=0.011)$ and only in one case hyperintense 
Fig. 1 STARD (Standards for Reporting of Diagnostic Accuracy) flow diagram of the study [2]

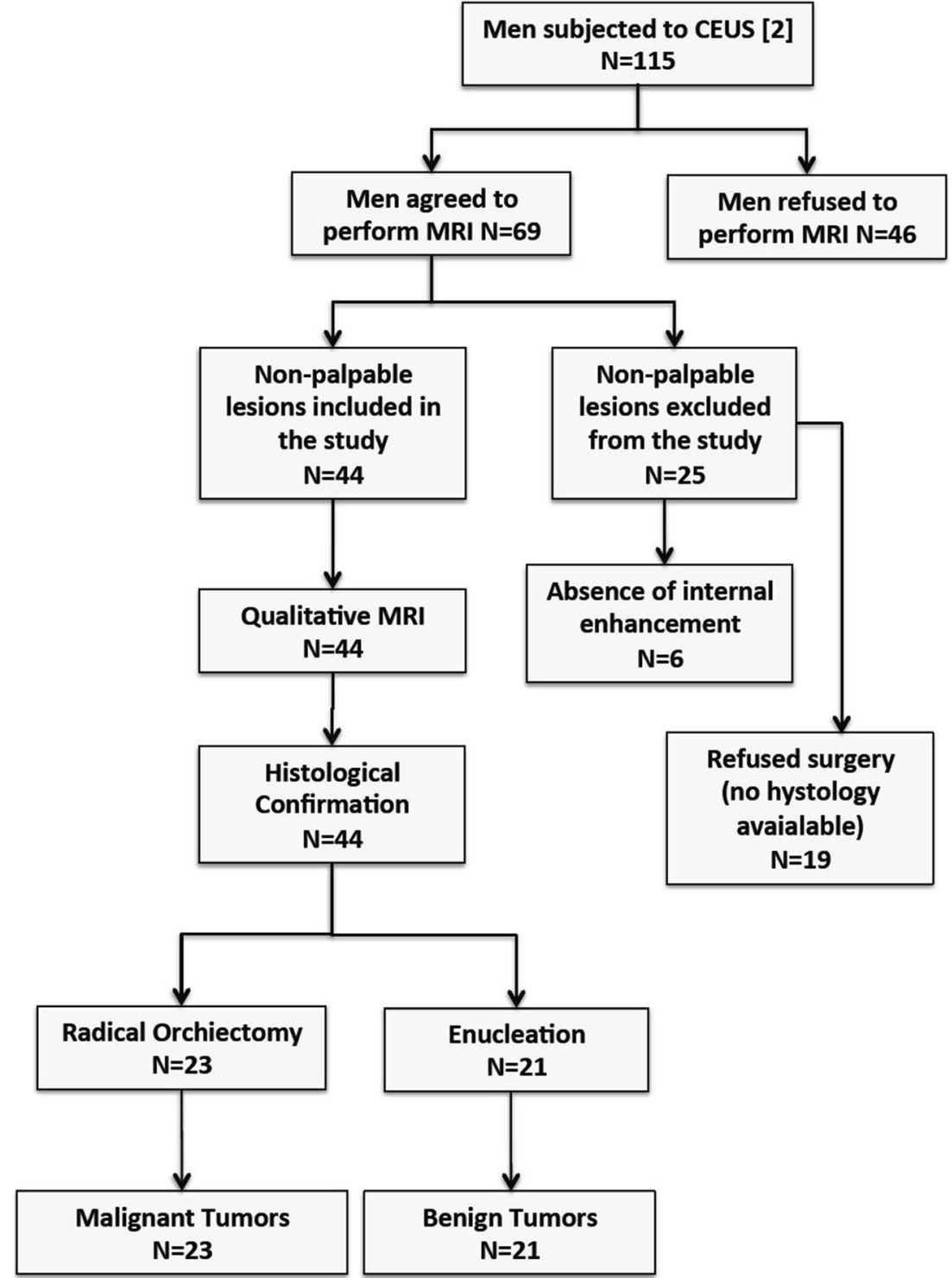

(one case of benign vs. one case of malignant lesions, $\chi 2$ $0.004, p=N$.S.). Well-defined margins were observed in 14 of the 21 benign lesions $(66.7 \%)$ compared with five of the 23 malignant lesions $(21.7 \%)\left(\chi^{2}\right.$ 9.031, $\left.\mathrm{p}=0.003\right)$. Blurred
Table 2 Clinical data (mean \pm standard deviation) of all patients and lesions according to the reference diagnosis

\begin{tabular}{|c|c|c|c|c|c|}
\hline \multirow[b]{2}{*}{ Final diagnosis } & \multirow[b]{2}{*}{$\mathrm{N}$} & \multirow[b]{2}{*}{ Age (y) } & \multirow[b]{2}{*}{ Diameter $(\mathrm{cm})$} & \multicolumn{2}{|c|}{ Testicular volume (mL) } \\
\hline & & & & $\mathrm{R}$ & $\mathrm{L}$ \\
\hline All patients & 44 & $34.45 \pm 8.97$ & $0.8 \pm 0.34$ & $12.6 \pm 5.05$ & $12.6 \pm 5.93$ \\
\hline Malignant tumours & 23 & $33.96 \pm 5.13$ & $0.9 \pm 0.31 *$ & $12.9 \pm 5.43$ & $12.6 \pm 5.68$ \\
\hline Pure seminoma & 20 & $33.57 \pm 5.84$ & $1.0 \pm 0.32$ & $10.50 \pm 4.65$ & $12.32 \pm 6.29$ \\
\hline Embryonal carcinoma & 2 & $29 \pm 4.24$ & $1.2 \pm 0.28$ & $19.2 \pm 3.18$ & $13.7 \pm 2.61$ \\
\hline Mixed germ cell tumour & 1 & 38 & 1.0 & 19.5 & 5.4 \\
\hline Benign tumours & 21 & $35 \pm 11.98$ & $0.6 \pm 0.28$ & $12.3 \pm 4.89$ & $12.6 \pm 6.30$ \\
\hline Leydig cell tumour & 19 & $36.0 \pm 13.91$ & $0.6 \pm 0.26$ & $12.9 \pm 4.95$ & $13.7 \pm 6.32$ \\
\hline Sertoli cell tumouur & 2 & $32.6 \pm 5.50$ & 0.4 & $11.9 \pm 2.96$ & $9.7 \pm 3.25$ \\
\hline
\end{tabular}

$* \mathrm{p}<0.001$ malignant tumours vs. benign tumours

$R$ right testis, $L$ left testis 
margins were more typical of malignant lesions (14/23, $61 \%$ vs. 5/21, 23.8, $\chi 26.145, \mathrm{p}=0.013$ ).

A homogeneous enhancement was observed predominantly in benign lesions $\left(18 / 21,85.7 \%\right.$ vs. $7 / 23,30.4 \%, \chi^{2}$ $13.672, \mathrm{p}<0.001)$, whereas rim enhancement was present only in malignant tumours $\left(4 / 23\right.$ vs. $\left.0 / 21, \chi^{2} 4.017, p=0.109\right)$.

\section{MRI semi-quantitative results}

Benign lesions (Fig. 2) had a more rapid and intense wash-in $\left(18 / 21,85.7 \%\right.$, vs. 5/23, $\left.21.7 \%, \chi^{2} 18.008, \mathrm{p}<0.001\right)$ and $\mathrm{a}$ prolonged wash-out $\left(15 / 21,71.4 \%\right.$ vs. $0 / 23, \chi^{2} 24.926, \mathrm{p}<$ 0.001), whereas malignant tumours (Figs. 3 and 4) showed a weak and progressive wash-in $(18 / 23,78.3 \%$ vs. $3 / 21, \chi 2$ $18.008, \mathrm{p}<0.001)$ and an absent wash-out $(21 / 23,91.3 \%$ vs. $6 / 21,28.6 \%, \chi^{2} 18.221, p<0.001$ ) (Tables 3 and 4). Subgroup comparisons of MRI features were also performed for the two most numerically representative histotypes: Leydig cell tumours (LCTs, 19 cases) and seminomas (20 cases). The results are summarized in Tables 3 and 4.

\section{Comparison between MRI and histology}

MRI findings led to a correct pre-operative characterization in $86 \%$ of tumours (39 of 44 cases): 17/21 of the benign and 22/ 23 of the malignant tumours. Of 18 lesions (41\%, 18/44) diagnosed as benign at MRI, 17 were found to be benign and one malignant at final histology. Of the 26 lesions (59 \%, 26/44) initially diagnosed as malignant at MRI, 22 were confirmed while four lesions turned out to be benign at final histology.

The sensitivity of scrotal MRI in diagnosing Leydig cell tumour was $89.47 \%$ (95 \% CI: 66.82- 98.39), and the specificity $95.65 \%$ (95\% CI: 77.98-99.27, Table 5).

The sensitivity of scrotal MRI in diagnosing malignant lesions was 95.65\% (95\% CI: 77.98-99.27), and the specificity $80.95 \%$ (95 \% CI: 58.08-94.44). The diagnostic accuracy was $93 \%$.

The Kappa value for the interobserver agreement obtained from the qualitative analysis was $0.854(\mathrm{p}<0.001$, CI $95 \%)$, characterizing a very good agreement between observers.

\section{Discussion}

Our study prospectively evaluated the accuracy of qualitative and semi-quantitative contrast-enhanced MRI in the differential diagnosis of non-palpable testicular masses. For the first time, we described that in a large prospective series of patients, Leydig cell tumour of the testis presents distinct MRI features that enable an accurate pre-operative diagnosis in $93 \%$ of the cases. Furthermore, our study provides a qualitative and semi-quantitative analytical description of gadolinium contrast kinetics in benign and malignant tumours, demonstrating typical features of Leydig cell tumours and further improving the differential diagnosis with seminomas.

US is considered the gold standard in the evaluation of scrotal pathology. Occasionally, during a routine US examination performed for other reasons, non-palpable testicular

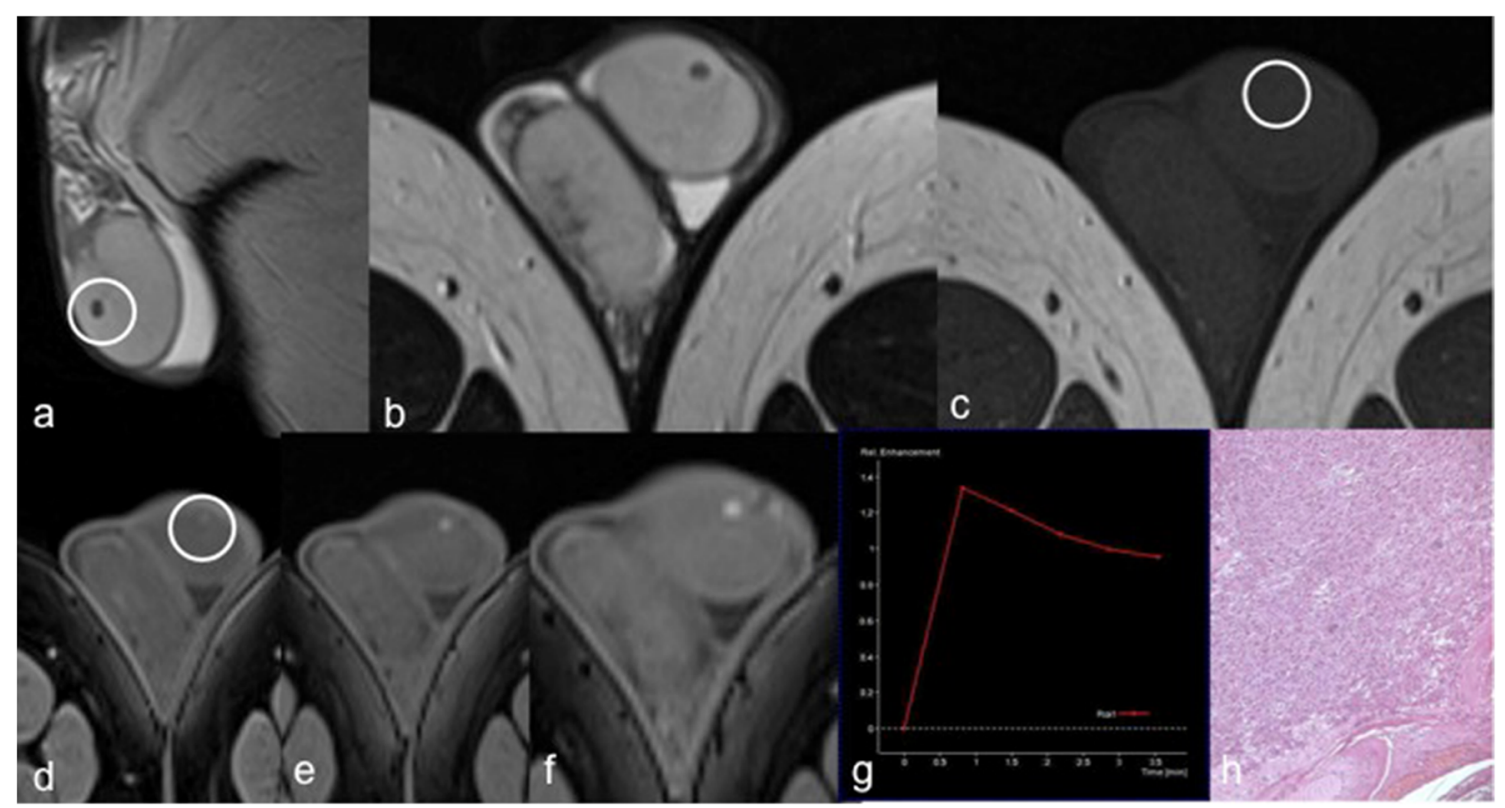

Fig. 2 Leydig cell tumour in the medium part of the left testes in a 38year-old man. T2 WI on the sagittal (a) and axial (b) planes: the lesion is well defined and markedly hypointense. Isointense on T1 WI (c). VIBE (volumetric interpolated breath-hold examination) subtracted T1 WI after contrast administration (d, e, f) shows a rapid and marked wash-in. The intensity-time curve (g) shows a peak within the first minute. Histological examination of the lesion (10x) with homogeneous tumour cells arranged in a row is shown in (h) 


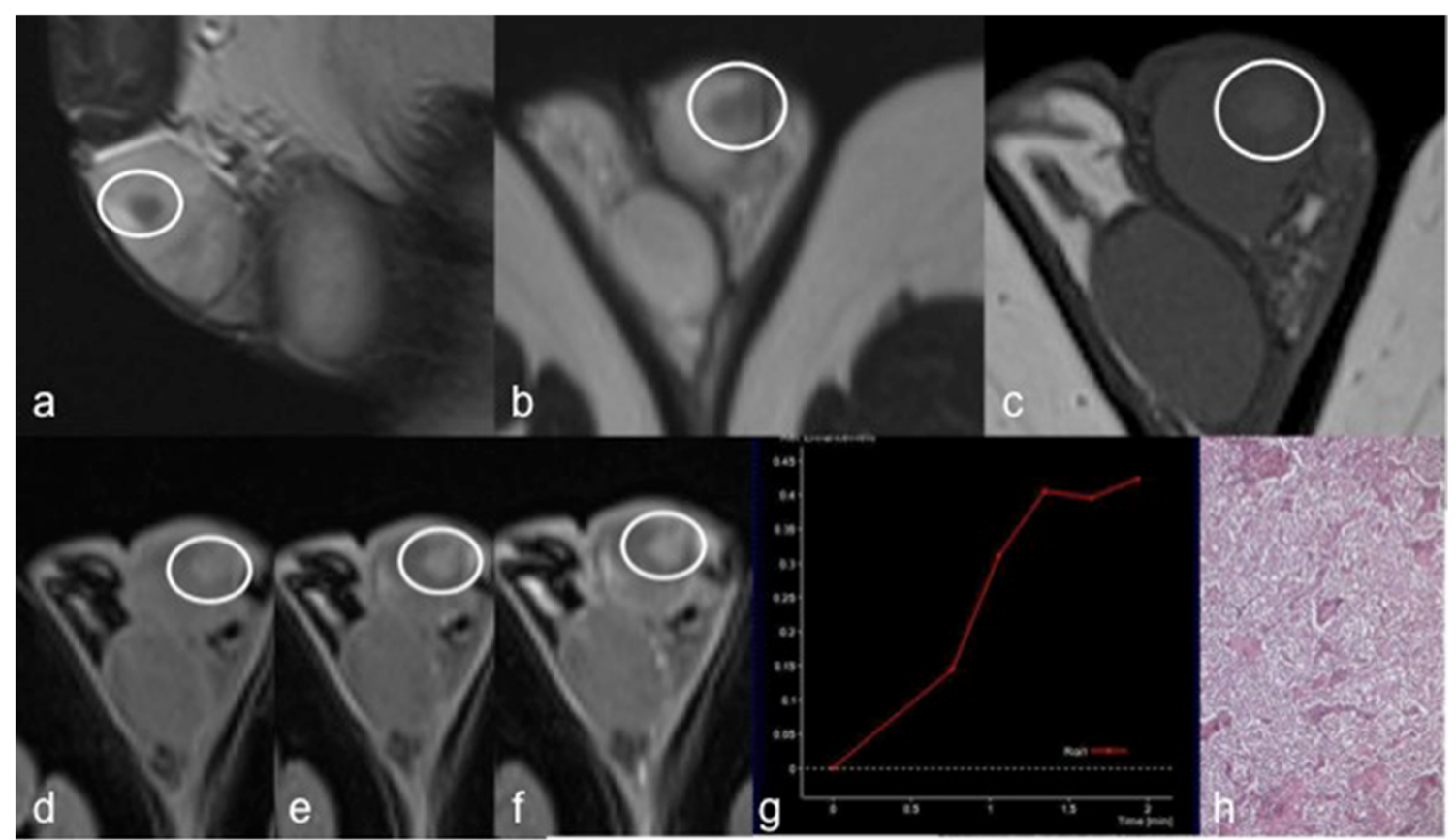

Fig. 3 Seminomatous lesion in the superior part of the left testes of a 35 year-old man. T2 WI on the sagittal (a) and axial (b) planes: the lesion appears slightly hypointense and with feathered margins. T1 WI on the axial plane shows a weak hyperintensity of the lesion (c). VIBE

lesions are identified; among these, approximately $10 \%$ are tumours, half of which are malignant [14-16].

US is able to identify a scrotal mass and to differentiate its origin (intra- or extratesticular) with a sensitivity range between $98 \%$ and $100 \%$ [17-19]. However, this imaging modality is considered insufficient in discriminating benign and malignant testicular neoplasms due to their frequent overlying appearance, particularly in case of smallest lesions.

Isidori et al. demonstrated that in experienced centres, CEUS can improve the diagnostic accuracy of unenhanced (volumetric interpolated breath-hold examination) subtracted T1 WI shows a weak and progressive enhancement $(\mathbf{d}, \mathbf{e}, \mathbf{f})$, which is well visible on the intensity-time curve $(\mathbf{g})$. Histological examination $(10 \mathrm{x})$ : the neoplasm contains abundant infiltration of lymphocytes (f)

US in the differential diagnosis of testicular non-palpable lesions [2, 20,21]. Similarly, the possibility of using MRI as the third level imaging in the study of testicular masses has been investigated, in particular if they are of difficult characterization [22].

Cramer et al. [23] demonstrated the value of MRI in the detection of testicular tumours; moreover, Adham et al. [24] found that it may be helpful in the histological characterization of testicular tumours. In addition, Schultz-Lampel et al. [25], evaluating 88 patients with clinical suspicion of testicular

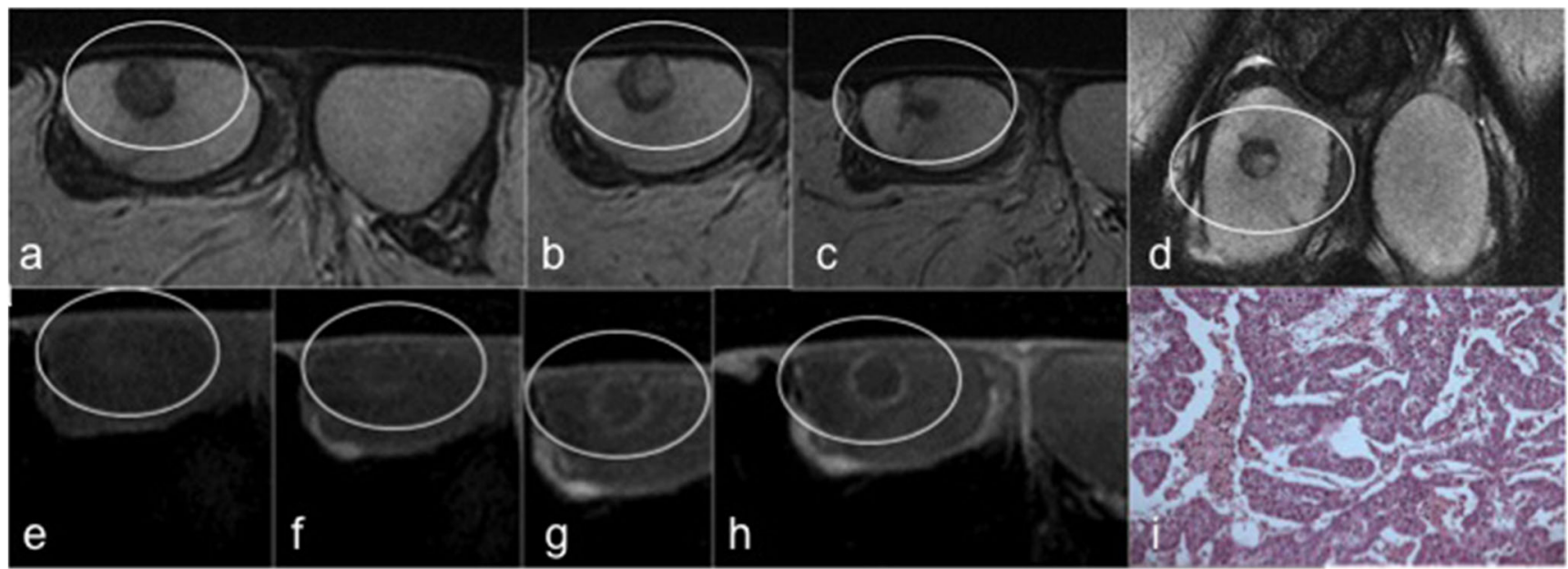

Fig. 4 Embryo cancer on the right testes of a 30-year-old man. (a, b, c) T2 WI. On a different axial plane, the lesion is non-homogeneous with a hypo-isointense rounded area. Four consecutive scans of post-contrast VIBE (volumetric interpolated breath-hold examination) sequences (e, $\mathbf{f}, \mathbf{g}, \mathbf{h})$ show the weak contrast enhancement only seen on the peripheral margin. Histological examination (10x) with atypical cells and necrotic areas (i) 
Table 3 Frequencies of qualitative features MRI. Data are presented as percentages (number)

\begin{tabular}{|c|c|c|c|c|c|c|c|c|}
\hline Modality & Feature & & $\begin{array}{l}\text { Benign tumour } \\
\mathrm{N}=21\end{array}$ & $\begin{array}{l}\text { Malignant tumour } \\
\mathrm{N}=23\end{array}$ & $\mathrm{p}$-value & $\begin{array}{l}\text { Leydig cell tumour } \\
\mathrm{N}=19\end{array}$ & $\begin{array}{l}\text { Seminomas } \\
\mathrm{N}=20\end{array}$ & $\mathrm{p}$ \\
\hline \multirow[t]{9}{*}{ MRI } & \multirow[t]{4}{*}{$\mathrm{T} 2-\mathrm{W}$} & Markedly hypointense & $81(17)$ & $34.8(8)$ & 0.003 & $89.4(17)$ & $35(7)$ & 0.002 \\
\hline & & Slightly hypointense & $14.3(3)$ & $52.2(12)$ & 0.012 & $5.3(1)$ & $60(12)$ & 0.003 \\
\hline & & Hyperintense & $4.8(1)$ & $4.3(1)$ & 0.948 & $5.3(1)$ & $5.0(1)$ & 0.055 \\
\hline & & Isointense & $0(0)$ & $8.7(2)$ & / & 1 & / & / \\
\hline & \multirow[t]{2}{*}{$\mathrm{T} 1-\mathrm{W}$} & Slightly hyperintense & $23.8(5)$ & $56.5(13)$ & 0.032 & $26.3(5)$ & $60(12)$ & 0.038 \\
\hline & & Isointense & $76.2(16)$ & $43.5(10)$ & 0.032 & $73.7(14)$ & $40(8)$ & 0.038 \\
\hline & \multirow[t]{3}{*}{ Margins } & Well defined & $66.7(14)$ & $21.7(5)$ & 0.004 & $68.4(13)$ & $10(2)$ & 0.001 \\
\hline & & Blurred & $23.8(5)$ & $60.9(14)$ & 0.016 & $26.3(5)$ & $70(14)$ & 0.009 \\
\hline & & Irregular & $9.5(2)$ & $17.4(4)$ & 0.454 & $5.3(1)$ & $20(4)$ & 0.198 \\
\hline \multirow[t]{8}{*}{ DCE-MRI } & \multirow[t]{3}{*}{ Enhancement } & Homogeneous & $85.7(18)$ & $30.4(7)$ & 0.001 & $94.7(18)$ & $30(6)$ & 0.001 \\
\hline & & Non-homogeneous & $14.3(3)$ & $52.2(12)$ & 0.012 & $5.3(1)$ & $60(12)$ & 0.003 \\
\hline & & Rim enhancement & $0(0)$ & $17.4(4)$ & 1 & $0(0)$ & $10(2)$ & 1 \\
\hline & \multirow[t]{2}{*}{ Wash-in } & Rapid and intense & $85.7(18)$ & $21.7(5)$ & $<0.001$ & $94.7(18)$ & $15(3)$ & $<0.001$ \\
\hline & & Weak and progressive & $14.3(3)$ & $78.3(18)$ & $<0.001$ & $5.3(1)$ & $85(17)$ & $<0.001$ \\
\hline & \multirow[t]{3}{*}{ Wash-out } & Slow and delayed & $71.4(15)$ & $0(0)$ & l & $78.9(15)$ & $0(0)$ & 1 \\
\hline & & Rapid & $0(0)$ & $8.7(2)$ & I & $0(0)$ & $10(2)$ & l \\
\hline & & Absent & $28.6(6)$ & $91.3(21)$ & $<0.001$ & $21.1(4)$ & $90(18)$ & $<0.001$ \\
\hline
\end{tabular}

cancer, reported that MRI was able to correctly characterize all cases, both malignant and benign, with the limitation, in that particular series, that nearly all benign lesions were attributable to paratesticular masses.
Tsili et al. [26] highlighted the parameters that mostly oriented towards characterization such as margins, signal intensity of T2-WI and, in a less significant way, of T1-WI, especially for lesions that are not definable as seminomas.

Table 4 Diagnostic performance of MRI in the differential diagnosis of testicular lesions

\begin{tabular}{|c|c|c|c|c|c|c|}
\hline \multirow[t]{2}{*}{ Modality } & & \multirow[t]{2}{*}{ Feature } & \multicolumn{2}{|c|}{ Benign vs. malignant tumours } & \multicolumn{2}{|c|}{ LCTs vs. seminomas } \\
\hline & & & $x^{2}$ & p-value & $x^{2}$ & \\
\hline \multirow[t]{9}{*}{ MRI } & \multirow[t]{4}{*}{$\mathrm{T} 2-\mathrm{W}$} & Markedly hypointense & 9.537 & $0.003^{\mathrm{a}}$ & 12.216 & $0.001^{\mathrm{a}}$ \\
\hline & & Slightly hypointense & 7.013 & $0.011^{\mathrm{a}}$ & 13.137 & $<0.001^{\mathrm{a}}$ \\
\hline & & Hyperintense & 0.004 & $1.000^{\mathrm{a}}$ & 0.001 & $1.000^{\mathrm{a}}$ \\
\hline & & Isointense & 1.913 & $0.489^{\mathrm{a}}$ & / & 1 \\
\hline & \multirow[t]{2}{*}{$\mathrm{T} 1-\mathrm{W}$} & Slightly hyperintense & 4.859 & 0.027 & 4.496 & 0.034 \\
\hline & & Isointense & 4.859 & 0.027 & 4.496 & 0.034 \\
\hline & \multirow[t]{3}{*}{ Margins } & Well defined & 9.031 & 0.003 & 14.050 & $<0.001^{\mathrm{a}}$ \\
\hline & & Blurred & 6.145 & 0.013 & 7.442 & 0.006 \\
\hline & & Irregular & 0.577 & 0.666 & 1.893 & $0.342^{\mathrm{a}}$ \\
\hline \multirow[t]{8}{*}{ DCE-MRI } & \multirow[t]{3}{*}{ Enhancement } & Homogeneous & 13.672 & $<0.001$ & 17.252 & $<0.001$ \\
\hline & & Non-homogeneous & 7.013 & 0.011 & 13.137 & $<0.001^{\mathrm{a}}$ \\
\hline & & Rim enhancement & 4.017 & 0.109 & 2.003 & $0.487^{\mathrm{a}}$ \\
\hline & \multirow[t]{2}{*}{ Wash-in } & Rapid and intense & 18.008 & $<0.001$ & 24.927 & $<0.001$ \\
\hline & & Weak and progressive & 18.008 & $<0.001$ & 24.927 & $<0.001$ \\
\hline & \multirow[t]{3}{*}{ Wash-out } & Slow and delayed & 24.926 & $<0.001$ & 25.658 & $<0.001^{\mathrm{a}}$ \\
\hline & & Rapid & 1.913 & 0.489 & 2.003 & $0.487^{\mathrm{a}}$ \\
\hline & & Absent & 18.221 & $<0.001$ & 18.837 & $<0.001^{\mathrm{a}}$ \\
\hline
\end{tabular}

${ }^{\text {a }}$ Fisher exact test

LCTs Leydig cell tumours 
Table 5 Diagnostic performance of MRI in diagnosing Leydig cell tumour
Diagnostic performance of MRI, \% $(95 \% \mathrm{CI})$

\begin{tabular}{lll}
\cline { 2 - 3 } & Diagnosing Leydig cell tumours & Diagnosing malignant lesions \\
\hline Sensitivity & $89.47(66.82-98.39)$ & $95.65(77.98-99.27)$ \\
Specificity & $95.65(77.98-99.27)$ & $80.95(58.08-94.44)$ \\
\hline
\end{tabular}

Diffusion-weighted imaging (DWI) and apparent diffusion coefficient (ADC) values are potentially valuable in the differentiation between normal, benign and malignant scrotal lesions, allowing complementary information for morphological and contrast-enhanced imaging. Qualitative evaluation of DWI may also be helpful, even if the quantitative ADC values are not useful.

According to others [27, 28], in our experience the wide individual variation of ADC measurements and the difficulty in suggesting the ADC cut-off value as an effective parameter for tissue characterization limited the use of DWI in the evaluation of testicular lesions. Our data confirm that a markedly hypointense signal on T2-WI is significantly associated with a benign nature of the lesion, and this feature may be suggestive of a Leydig cell tumour $(80 \%$ of all benign and $90 \%$ of Leydig cell tumours included in our series). Furthermore, a typical pattern of contrast enhancement, characterized by homogeneous impregnation $(p=0.001)$ and rapid and marked wash-in $(\mathrm{p}<0.001)$, was found in $94.7 \%$ of Leydig cell tumours, accompanied by a slow and late wash-out $(\mathrm{p}<0.001)$. This behaviour, with the appropriate distinction, is similar to what was observed in contrast-enhanced US [2], and has been attributed to a high degree of vascularization of Leydig cell tumours, local estrogen generation and an increased level of endocrine-gland vascular endothelial growth factor (EG-VEGF) expressed by Leydig cells [29-31]. Malignant lesions were significantly associated with blurred margins $(p=0.013)$ and a weak hypointense signal on T2-WI $(p=0.011)$. Seminomas showed this feature in $70 \%(p=0.009)$ and $60 \%(p=0.003)$ of the cases, respectively. Another feature found in this study is represented by a weak signal hyperintensity on $\mathrm{T} 1$ in $60 \%$ of cases of seminoma $(\mathrm{p}=0.038)$. Furthermore, seminomatous lesions showed weak and progressive wash-in $(p<0.001)$ in $85 \%$ of cases, with an absent wash-out $(\mathrm{p}<0.001)$. Our pattern differs from those reported in other studies in which seminomas are characterized by an enhancement with a rapid increase of signal intensity followed by gradual washout of the contrast medium [12]. The Sertoli cell tumours in our series showed a DCE-MRI behaviour similar to seminomas, and therefore were erroneously considered malignancies. Because of their rarity, a conclusive statement cannot be made; however, recent data suggest that Sertoli cell tumours do not express EG-VEGF to the high level seen in LCTs [29]. An important finding of this study was the very high frequency of Leydig cell tumours. This supports the hypothesis that Leydig cell dysfunction has a role in testicular dysgenesis syndrome [28] and it is sustained by the finding of subclinical hormonal failure (high $\mathrm{LH}$ or reduced testosterone) found in $20.5 \%$ and $13 \%$ of our patients, respectively [32].

Our study has some limitations: first, our series included patients with small $(<1.5 \mathrm{~cm})$ non-palpable lesions evaluated by CEUS in a previous study [2], which already, and independently of MRI findings, improved the differential diagnosis and the individualized management of small testicular lesions. Despite these results, MRI was performed blinded to CEUS results to evaluate prospectively the accuracy of qualitative and semiquantitative contrast-enhanced MRI in the differential diagnosis of non-palpable testicular masses. Secondly, the semi-quantitative analysis, which depends on the placement of the ROI, could be affected by the small size of the tumour. Third, we can't ignore that the case mix may have affected the sensitivity (one falsenegative case) and the specificity (four false-positive cases) of MRI. We considered four benign tumours to be malignant (two Sertoli cell tumours and two LCTs) because they showed two features that we attributed to malignant neoplasms: weak and progressive wash-in, absent wash-out and a slight hypointense signal on T2weighted images. Nevertheless, to our knowledge the present series is the largest prospective collection of MRI imaging of Leydig cell tumours. The only falsenegative case of the study (one embryonal carcinoma) was misdiagnosed because of its well-defined margins and rim enhancement, which had made radiologists lean towards the nature of an active inflammation, supported by the clinical history of the patient who reported fever, elevated white cell count, pain in the scrotal region and negative markers. In our sample, both cases of embryonal carcinomas showed a pattern of rim enhancement, requiring a differential diagnosis toward inflammatory lesions. Even if this feature cannot be standardized, it may find an explanation in the aggressive nature of this tumour, which is often characterized by areas of haemorrhage and necrosis, producing a central non-enhancing 
area; moreover, in agreement with our data [33, 34], even at CEUS, embryonal carcinomas were characterized by poor or absent internal contrast enhancement [15].

In conclusion, the results achieved with our protocol (further improvements can be obtained by use of specified perfusion sequences and 3D high resolution T2 sequences), demonstrated that MRI might be proposed in addition to unenhanced US and CEUS when it is necessary to decide the best therapeutic procedure for a testicular mass not yet characterized. Our study demonstrated that MRI is able not only to characterize benign and malignant testicular lesions, with a diagnostic accuracy of $93 \%$, but also suggests a specific diagnostic hypothesis when specific imaging features are present. Well-defined margins, a markedly hypointense signal on T2-WI, and a rapid and marked wash-in followed by a slow and late wash-out, should orient toward the diagnosis of Leydig cell tumour. On the other hand, blurred margins, a slightly hypointense signal on T2-WI and a slightly hyperintense signal on T1-WI, associated with a weak and progressive wash-in followed by an absent wash-out, are highly specific for the diagnosis of seminoma.

Acknowledgments The scientific guarantor of this publication is Lucia Manganaro. The authors of this manuscript declare no relationships with any companies whose products or services may be related to the subject matter of the article. The authors state that this work has not received any funding. One of the authors has significant statistical expertise. Institutional Review Board approval was obtained. Written informed consent was obtained from all subjects (patients) in this study. Some study subjects or cohorts have been reported in a previous CEUS study by Isidori et al. (Radiol 273(2):606-18) conducted in parallel with our study.

Methodology: prospective, diagnostic study, performed at one institution.

\section{References}

1. La Vecchia C, Bosetti C, Lucchini F et al (2010) Cancer Mortality in Europe, 2000-2004, and an overview of trends since 1995. Ann Oncol 21(6):1323-1360

2. Isidori A, Pozza C, Gianfrilli et al (2014) Differential diagnosis of non-palpable testicular lesions: qualitative and quantitative contrast enhanced ultrasound of benign and malignant testicular tumours. Radiology 273(2):606-618

3. Carmignani L, Colombo R, Gadda F et al (2007) Conservative surgical therapy for Leydig cell tumour. J Urol 178(2):507-511

4. Giannarini G, Dieckmann KP, Abers P et al (2010) Organ sparing surgery for adult testicular tumours: a systemaic review of the literature. Eur Urol 57(5):780-790

5. Dogra VS, Gottlieb RH, Oka M et al (2003) Sonography of the scrotum. Radiology 227(18-36):31

6. Sohaib SA, Koh DW, Husband JE (2008) The role of imaging in the diagnosis, staging and management of testicular cancer. AJR 191: 387-395

7. Lock G, Schröder C, Schmidt C et al (2014) Contrast-Enhanced Ultrasound and Real-Time Elastography for the Diagnosis of Benign Leydig Cell Tumours of the Testis - A Single Center Report on 13 Cases. Ultraschall Med 35(6):534-539
8. Huang DY, Sidhu F (2012) Focal testicular lesions: colour Doppler ultrasound, contrast-enhanced ultrasound and tissue elastography as adjuvants to the diagnosis. Br J Radiol 85(1):41-53

9. Cantisani V, Olive M, Di Segni M et al (2012) Contrast-enhanced ultrasonographic (CEUS) and elastosonographic features of a case of testicular Leydig tumour. Ultraschall Med 33(5):407-409

10. Kim W, Rosen MA, Langer JE et al (2007) US-MR Imaging correlation in pathologic conditions of the scrotum. Radiographics 27(5):1239-1253

11. Watanabe Y, Dohke M, Ohkudo K et al (2000) Scrotal disorders: evaluation of testicular enhancement patterns at dynamic contrastenhanced subtraction MR imaging. Radiology 217:219-227

12. Tsili AC, Argyropoulou MI, Astrakas LG et al (2013) Dynamic contrast-enhanced subtraction MRI for characterizing intratesticular mass lesions. AJR 200:578-585

13. Fleiss JL, Kingman A (1990) Statistical management of data in clinical research. Crit Rev Oral Biol Med 1:55-66

14. Isidori AM, Lenzi A (2008) Scrotal Ultrasound: Morphological and Functional Atlas. Editore Accademia Nazionale di Medicina 19:49

15. Schill W. B. et al. (2010) Andrologia clinica. Traduzione a cura di Lenzi A. ed Isidori A.M. Springer-Verlag Italia, p.183

16. Schmoll HJ et al (2004) European consensus on diagnosis and treatment of germ cell cancer: a report of European Germ Cell Cancer Consensus Group (EGCCCG). Ann Oncol 15:1377-1399

17. Schwerk WB, Schwerk WN, Rodeck G (1987) Testicular tumours: prospective analysis of real-time US patterns and abdominal staging. Radiology 164:369-374

18. Rifkin MD, Kurtz AB, Pasto ME et al (1985) Diagnostic capabilities of highresolution scrotal ultrasonography: prospective evaluation. J Ultrasound Med 4:13-19

19. Benson CB, Doubilet PM, Richie JP (1989) Sonography of the male genital tract. AJR Am J Roentgenol 153:705-713

20. Lock G, Schmidt C, Helmich F et al (2011) Early experience with contrast-enhanced ultrasound in the diagnosis of testicular masses: a feasibility study. Urology 77(5):1049-1053

21. Shah A, Lung PF, Clarke JL et al (2010) Re: New ultrasound techniques for imaging of the indeterminate testicular lesion may avoid surgery completely. Clin Radiol 65:496-497

22. Muglia V, Tucci S, Elias J et al (2002) Magnetic resonance imaging of scrotal diseases: when it makes the difference. Urology 59:419 423

23. Cramer BM, Schlegel EA, Thueroff JW (1991) MR imaging in the differential diagnosis of scrotal and testicular disease. RadioGraphics 11:9-21

24. Adham WK, Raval BK, Uzquiano MC et al (2005) Best Cases from the AFIP: Bilateral Testicular Tumors: Seminoma and Mixed Germ Cell Tumor. Radiographics 25:835-839

25. Schultz-Lampel D, Bogaert G et al (1991) (1991) MRI for evaluation of scrotal pathology. Urol Res 19:289-292

26. Tsili AC, Tsampoulas C, Giannakopoulos X et al (2007) MRI in the histologic characterization of testicular neoplasms. AJR 189:1473

27. Algebally AM, Tantawy HI, Yousef RR (2014) Value of diffusion weighted magnetic resonance imaging in diagnosis and characterization of scrotal abnormalities. Egypt J Radiol Nucl Med 45(3): 949-958

28. Tsili AC, Argyropoulou MI, Giannakis D et al (2012) Diffusionweighted MR imaging of normal and abnormal scrotum: preliminary results. Asian J Androl 14(4):649-654

29. Samson M, Peale FV Jr, Frantz G et al (2004) Human endocrine gland-derived vascular endothelial growth factor: expression early in development and in Leydig cell tumors suggests roles in normal and pathological testis angiogenesis. J Clin Endocrinol Metab 89(8):4078-4088

30. Kilic N, Lauke H, Fiedler W et al (1999) Angiogenic switch and vascular stability in human Leydig cell tumours. Angiogenesis 3(3): $231-240$ 
31. Fernández GC, Tardáguila F, Rivas C et al (2004) Case report: MRI in the diagnosis of testicular Leydig cell tumour. Br J Radiol 77(918):521-524

32. Giannetta E, Gianfrilli D, Barbagallo F (2012) Subclinical male hypogonadism. Best Pract Res Clin Endocrinol Metab 26(4):539550
33. Rosai J (2004) Rosai and Ackerman's surgical pathology, 9th ed., vol. 1. Elsevier, Philadelphia, PA

34. Ulbright TM, Amin MB, Young RH (1997) Atlas of tumor pathology: tumors of the testis, adnexa, spermatic cord, and scrotum. 3rd ser, fasc 25. Armed Forces Institute of Pathology, Washington, pp 59-191 